\title{
OLIGOPOLIO Y TIPOS DE INTERÉS EN LA BANCA ESPAÑOLA, 1942-1975 *
}

\section{M. ${ }^{a}$ ÁNGELES PONS BRIAS}

\author{
Universidad de Valencia
}

\section{RESUMEN}

El objetivo de este trabajo es analizar la posible utilización por parte de los bancos españoles de acuerdos monopolistas destinados a fijar los tipos de interés en el periodo comprendido entre el final de la guerra civil española y 1975. El segundo aspecto que va a abordar este artículo es la búsqueda de indicadores cuantitativos y cualitativos del grado de efectividad de dichos acuerdos colusivos. La principal conclusión que se obtiene es que a pesar de la existencia de un pacto entre las entidades bancarias para controlar los tipos de interés, respaldado por las propias autoridades franquistas, los principales bancos infringieron dichos acuerdos y compitieron vía precio en el mercado de captación de depósitos, especialmente a partir de 1950.

$N$ de E. Fecha de recepción del artículo en la Revista de Historia Económica: septiembre de 1999.

Fecha de aprobación por el Consejo de Redacción: febrero de 2001.

Quiero agradecer a Teresa Tortella y al personal del Archivo Histórico del Banco de España la ayuda prestada en la consulta de un material que estaba pendiente de catalogar. Debo agradecer también la ayuda prestada por el personal del Archivo del Banco de Inglaterra. Asimismo, quiero dar las gracias a José Luis García Ruiz por las observaciones y sugerencias que realizó a una versión anterior de este trabajo, así como por la información que tanto él como Gabriel Tortella tuvieron la amabilidad de suministrarme. Agradezco los comentarios realizados por los miembros del Departamento de Historia Económica de la Universidad Carlos III, la Universidad Pompeu Fabra y el Departamento de Análisis Económico de la Universidad de Valencia en distintos seminarios impartidos en dichas universidades

Revista de Historia Económica 


\section{ABSTRACT}

This paper discusses the price collusive behaviour of the main Spanish banks from the end of the Civil War to 1975. The second purpose of this paper is to obtain quantitative and qualitative indicators of the degree of effectiveness of these cartel agreements. I conclude that in spite of the existence of collusive pacts to fix price conditions, the main banks broke cartel agreements and they competed in prices in the deposit market side, and especially from the mid-1950s onward.

\section{INTRODUCCIÓN}

El análisis sobre el nivel de competencia de la banca española desde el final de la guerra civil hasta mediados de los setenta se ha basado esencialmente en el estudio de tres variables: el nivel de concentración del sistema bancario ${ }^{1}$, la rentabilidad del sector ${ }^{2}$ y las conexiones existentes entre las distintas entidades bancarias a través de miembros comunes en sus consejos de administración ${ }^{3}$. Las principales conclusiones que se extraen de dichos trabajos son dos. En primer lugar, que la banca española tuvo un elevado nivel de concentración durante este período, siendo dicho grado de concentración un indicador del poder de mercado del sector. En segundo lugar, que gracias a su poder monopolista, logrado en gran medida gracias a una legislación muy proteccionista, los bancos consiguieron reducir el nivel de competencia y obtener unos sustanciales beneficios. Menor atención, sin embargo, se ha prestado al comportamiento de los precios, es decir, a la evolución de los tipos de interés de los depósitos y de los créditos aplicados por estas entidades. El examen de la conducta de los bancos en lo que a fijación de precios se refiere, así como la posible adopción de acuerdos colusivos entre las entidades bancarias ofrecería, sin embargo, una información muy útil sobre el nivel de competencia del sistema bancario español en este período. La ausencia de estudios sobre esta cuestión es una consecuencia de la escasa información disponible, ya que el acceso a los archivos privados de los principales bancos es muy limitado. Pese a las dificultades existentes, el objetivo de este trabajo es doble. En primer lugar, analizar si existe alguna evidencia sobre la uti-

' Véase, por ejemplo, De la Sierra (1953), Muñoz (1969), Fanjul y Maravall (1985) o Caminal, Gual y Vives (1990).

2 Véase Muñoz (1969) o Fanjul y Maravall (1985).

3 Véase De la Sierra (1953) o Muñoz (1969). 
lización de prácticas restrictivas de la competencia por parte de los bancos españoles en el período antes mencionado. En segundo lugar, en el caso en que dichos acuerdos monopolísticos hubiesen existido, obtener algunos indicadores sobre el nivel de cumplimiento de los mismos.

Para poder evaluar la importancia de este trabajo, es necesario analizar en qué medida los estudios tradicionales a los que hemos hecho referencia anteriormente ofrecen una interpretación adecuada sobre cuál fue el comportamiento de la banca española en la etapa franquista. La existencia de barreras de entrada y, en general, de un marco legislativo proteccionista impidió un funcionamiento competitivo del sector. El problema es cómo medir esa falta de competencia. Investigaciones muy recientes muestran que algunos de los argumentos que se han utilizado de forma reiterada para definir a la banca española como un sector monopolista son discutibles. Respecto a las relaciones entre las entidades bancarias y la influencia de las mismas en los principales grupos industriales, el trabajo pionero de De la Sierra (1957) y el más elaborado de Muñoz (1968) demuestran con claridad tanto las fuertes conexiones existentes entre los bancos españoles como los vínculos entre los principales bancos y las grandes empresas del país. Sin embargo, es muy difícil evaluar la influencia real de dichas conexiones, que indiscutiblemente existieron, en el nivel de competencia del sector. La banca alemana, por ejemplo, se ha caracterizado por tener fuertes vínculos con las empresas industriales y, sin embargo, este hecho por sí solo no es suficiente para considerar a dicho sector bancario como no competitivo (Tilly, 1993). La literatura ha hecho especial hincapié en los aspectos negativos que puede tener la participación de la banca en las empresas industriales en términos de aumento de poder y restricción de la competencia. Recientes estudios, sin embargo, muestran cómo la presencia de miembros de las entidades bancarias en los consejos de administración de las principales empresas industriales puede tener efectos positivos. En particular, estas relaciones permitirían reducir los problemas de información asimétrica asociados a la actividad de intermediación y fomentar las relaciones de largo plazo entre las empresas y los bancos (Edwards y Fischer, 1994).

Respecto a la obtención por parte de la gran banca de beneficios extraordinarios, los trabajos de Tafunell (1998) y Pons (2001) indican que si bien desde 1946 la rentabilidad bancaria estuvo por encima de la rentabilidad media obtenida para una muestra de grandes empresas industriales, las diferencias no fueron muy significativas. Si tenemos en cuenta que, siguiendo a Martínez Méndez (1991), la distinta naturaleza de los negocios afecta 
a la cifra de rentabilidad y que, por tanto, debemos esperar que las empresas financieras presenten una mayor tasa de rentabilidad que las no financieras, la obtención por parte de los bancos españoles de beneficios extraordinarios es cuestionable. Es más, el margen entre la rentabilidad bancaria y la rentabilidad de las empresas industriales se amplía desde 1970, precisamente en el período en el que se inicia la liberalización del sistema bancario español.

Una parte considerable de los beneficios de los grandes bancos espanooles procedía no de la actividad de intermediación sino de la obtención de plusvalías en las emisiones de capital. Los grandes bancos obtuvieron ganancias importantes a través de esta vía (Muñoz, 1969), lo que indicaría que si sumamos los beneficios derivados de la actividad de intermediación a las plusvalías, obtendríamos unos resultados muy elevados para la banca española. En cualquier caso, los aumentos más importantes de plusvalías se consiguen a partir de los años sesenta y no anteriormente.

Finalmente, otra de las variables que se ha utilizado para demostrar el poder de monopolio de la banca española es el nivel de concentración. Como demuestra Pueyo (1998), si bien es cierto que desde el final de la guerra civil hasta mediados de los cincuenta se produjo un aumento de la concentración del sistema bancario español, desde 1954 la concentración del sector -así como la de los grupos bancarios- disminuye. Sería muy tentador utilizar la evolución de este indicador como una muestra del debilitamiento del carácter oligopolista de la banca española, ya que el denominado paradigma «estructura-conducta-funcionamiento» identifica nivel de concentración con poder de mercado (Weiss, 1991). Sin embargo, el número de empresas que componen una industria depende de cuál es la estructura de costes de dicha industria. Por tanto, una mayor concentración en un determinado sector puede ser un indicador de la utilización de acuerdos colusivos, o de que existen economías de escala. Así pues, lo que estos datos ponen de manifiesto es que el nivel de concentración no es un buen indicador del grado de competencia de un sector (Smirlock, 1981).

La principal conclusión que se obtiene es que existen todavía muchos interrogantes sobre el funcionamiento del sistema bancario español durante la etapa franquista. Por tanto, es necesario proceder a un análisis más detallado de las prácticas bancarias durante este período que nos permita observar si, partiendo de la evidencia de que el sistema bancario español no funcionó competitivamente, la etapa 1939-1975 es un periodo homogéneo o si, por el contrario, se produjeron cambios significativos a lo largo 
de estos años que afectaron al nivel de competencia del sector. En esta línea se enmarca este trabajo, que, como he señalado anteriormente, pretende obtener información sobre la conducta de los principales bancos en la determinación de los tipos de interés.

\section{EL MARCO LEGISLATTVO}

Al finalizar la guerra civil española, el gobierno franquista impuso un marco legislativo para el sector bancario muy restrictivo tanto en términos de barreras de entrada, como de expansión de las entidades bancarias a través de la creación de nuevas oficinas, e incluso sobre las operaciones bancarias. En lo que a tipos de interés se refiere, la Orden Ministerial (OM) de 25 de noviembre de 1938 fijaba los tipos de interés máximos de las operaciones pasivas, mientras que la OM de 24 de julio de 1941 establecía las tarifas mínimas obligatorias de las operaciones activas y pasivas, sustituyendo las establecidas en enero de 1936.

En 1946, cuando vencía el plazo de veinticinco años fijado en la Ley de Ordenación Bancaria (LOB) de 1921 para la prórroga del privilegio de emisión de billetes a favor del Banco de España, se promulgó una nueva $L O B$ que ratificó gran parte de las medidas adoptadas en los años de la posguerra. La LOB de 1946 estableció los principios que iban a guiar a la economía española en materia financiera y bancaria hasta mediados de los sesenta. En lo referente a tipos de interés, el Proyecto de LOB de 1946 en su artículo 41 establecía que el Ministro de Hacienda, previo informe del Consejo Superior Bancario (CSB), podía fijar los tipos de interés y comisiones en las operaciones bancarias y las condiciones de su aplicación. El principal objetivo de esta medida era establecer tipos de interés bajos que favoreciesen la recuperación de la economía española.

La reacción de las entidades bancarias ante dicho Proyecto de Ley puede observarse a través de los informes que el CSB y algunos bancos (en concreto el Banco Vizcaya) realizaron sobre dicho proyecto para posteriormente remitirlos al Ministerio de Hacienda (AHBE, 1946a; AHBE, 1946b). En ambos informes se señala en su primer párrafo que «el proyecto todo responde a un criterio de intensificación del intervencionismo estatal, no sólo en lo que afecta al banco emisor, sino también a la banca privada». En ese sentido, el informe señala la necesidad de disminuir el grado de intervención del Estado en las actividades bancarias. En lo que respecta a la política de tipos de interés, el memorándum solicitaba modificar el 
artículo 41 del Proyecto de Ley. Hasta ese momento, y desde 1927, año en el que se establecieron las denominadas «Tarifas de Condiciones Mínimas», la intervención del Estado se había traducido en señalar los topes máximos y mínimos en las cuentas acreedoras y deudoras respectivamente. Por tanto, una fijación específica y concreta de los tipos por parte del gobierno era vista por las entidades bancarias como «un exceso manifiesto que no ha podido estar en la mente de los autores del proyecto» (AHBE, 1946a). Es más, el memorándum, de forma un tanto irónica, señala que no cree que haya estado en la intención de los legisladores interferir de forma tan grave en la actividad de los bancos privados y que, por tanto, es posible que no se trate más que «de una errata material» en la redacción del Proyecto de Ley.

$\mathrm{El}$ informe presentado por el CSB propone introducir una enmienda al artículo 41, para que en su redacción final se establezca la capacidad del Ministro de Hacienda de fijar los tipos de interés máximos de las operaciones pasivas y los tipos de interés mínimos de las operaciones bancarias activas. En su opinión, esta medida favorecería al funcionamiento del sistema bancario y de la economía en general por dos motivos. En primer lugar, porque evitaría un aumento excesivo de la competencia entre los bancos para atraer los fondos de los ahorradores y, por tanto, impediría un crecimiento desmesurado de los tipos de interés, cuando «la tónica general debe ser la del abaratamiento del dinero». En segundo lugar, dicho informe señalaba que el establecimiento de tipos de interés mínimos para las operaciones activas evitaría que como consecuencia de un aumento de la competencia se estableciesen tipos de interés sobre los créditos tan bajos que no permitiesen cubrir los costes. Por tanto, un tipo de interés mínimo sobre las operaciones activas limitaría la utilización de prácticas demasiado arriesgadas por parte de los bancos que podría llevarles incluso a la bancarrota. Lo que el informe muestra, por tanto, es una oposición clara a la competencia vía precios entre las entidades.

En la redacción final de la LOB de 1946 se recogió la petición formulada a través del CSB por parte de la banca privada y se estableció en el artículo 43 que el Ministro de Hacienda, previo informe del Banco de España, del CSB, de la Organización Sindical y del Consejo de Economía Nacional, podía fijar los tipos de interés máximos a las operaciones pasivas y los tipos y comisiones mínimas a las operaciones activas. Como ya reconocía Paris Eguilaz (1947), con este marco legal no se impedía la utilización de prácticas monopolistas por parte de las entidades bancarias españolas. Su opinión es relevante si tenemos en cuenta que Paris Eguilaz 
era en esos momentos Presidente del Consejo de Economía Nacional, órgano que según la misma Ley debía ser consultado para la fijación de los tipos de interés. Por tanto, al establecer dicha medida las autoridades franquistas eran conscientes de que dejaban en manos de la banca privada la posibilidad de establecer acuerdos monopolísticos para determinar los tipos de interés de los depósitos y créditos.

La legislación sobre tipos de interés no sufre modificaciones hasta mediados de los sesenta. La OM de 30 del octubre de 1964 fijó unos tipos de interés sobre operaciones pasivas inferiores a los vigentes en virtud de la OM de 25 de noviembre de 1938 (reducidos en la práctica por acuerdos interbancarios). A través de esta $O M$ se fijan por primera vez limitaciones de tipos de interés sobre operaciones activas, cuyo carácter no se precisa, de donde se deduciría el de tipos únicos, salvo en determinados casos a los que se otorgaba carácter de mínimos (Martínez Méndez, 1991). Se reformaron las tarifas de comisiones sobre operaciones activas y pasivas, con el carácter, en todos los casos, de mínimas. Finalmente, la Ley 13, de 18 de marzo de 1966, facultaba al Ministro de Hacienda, previo informe del Banco de España y del CSB, a señalar los tipos de interés máximos a las operaciones pasivas y tipos máximos y mínimos a las operaciones activas.

La principal diferencia de la legislación sobre tipos de interés desde mediados de los sesenta con respecto a la anterior es que se produjo una mayor variación de los tipos máximos y mínimos. Por ejemplo, si bien los tipos de interés de los depósitos se mantuvieron inalterados desde 1939 hasta 1964, con posterioridad hay ligeras modificaciones en los mismos en 1967, 1969, 1970, 1973 y 1974.

En un sistema bancario monopolista los bancos trataran de pagar un tipo de interés sobre sus depósitos por debajo del de equilibrio, mientras que cobrarán el máximo tipo de interés posible sobre sus créditos. La conclusión que obtenemos tras analizar el marco legislativo existente desde 1939 hasta mediados de los sesenta es que la regulación bancaria permitía a priori a los bancos españoles establecer acuerdos monopolísticos para fijar los precios.

\section{EVIDENCIA EMPÍRICA}

Dado que el marco legislativo no impedía la utilización de prácticas de colusión, en este apartado examinaremos si existe evidencia que demues- 
tre que los bancos españoles durante este período llegaron a algún acuerdo de este tipo. El CSB (1952) hace referencia a la existencia de un compromiso entre los bancos privados para fijar un tipo de interés sobre las operaciones pasivas por debajo del máximo legal y un tipo de interés sobre las operaciones activas por encima del mínimo legal. El CSB denomina a dichos acuerdos «Ordenación Reguladora de Condiciones Mínimas». El Ministerio de Hacienda no sólo autorizó la aplicación del convenio sino que permitió la creación de dos organismos (la Junta de Vigilancia y la Junta Superior) cuyo principal objetivo era velar por el cumplimiento de dichos acuerdos y sancionar las posibles infracciones. Aquellos bancos que decidiesen no participar en estos pactos se verían discriminados con respecto a los miembros que aceptasen el acuerdo (CSB, 1953).

Es difícil saber si este tipo de acuerdos para fijar los tipos de interés existieron antes de la guerra civil. Sin embargo, como señalan Tortella y García Ruiz (1993), la ausencia de competencia entre las entidades financieras a través de los tipos de interés se remontaría al artículo 10 de la LOB de 1921, en el que se favorecía la unificación de los tipos de interés bajo la supervisión del CSB. La primera referencia que se puede encontrar sobre la utilización de prácticas monopolistas para fijar los tipos de interés aparece en la revista España Económica y Financiera de 19 de noviembre de 1949 (Tortella y García Ruiz, 1993). No existe evidencia empírica sobre la existencia de pactos similares antes de ese año. Velarde (1961) cita la existencia de lo que él denomina un «Arreglo Moderador de la Competencia Bancaria». Aunque no indica de forma explícita cuáles son los términos en los que se realizó dicho acuerdo, el autor explica cómo en 1949 , al crecer la lucha entre los bancos por aumentar sus cuentas acreedoras, se llegó a este pacto. Aunque el CSB (1949) señala que algunos bancos infringieron estos compromisos, considera que las infracciones fueron menores. A partir de 1954 el CSB no vuelve a hacer ninguna referencia a este tipo de acuerdos.

A principios de los setenta, los principales bancos españoles crearon el denominado por la prensa «Club de los Siete Grandes», que reunía semanalmente a los siete mayores bancos con el gobernador del Banco de España para coordinar sus acciones. Tortella y García Ruiz (1999), consideran que fue Ignacio Vllalonga, presidente del Banco Central, quien en 1962 reunió a los banqueros en unas comidas mensuales que a partir de 1971 se harían semanales y bajo la presidencia del Banesto. Sin embargo, desde mediados de los setenta dicho club empezó a disolverse y, como señalan Tortella y García Ruiz (1993), la información suministrada por 
el CSB sobre tipos de interés declarados para el período 1968-1975 muestra dos cosas. En primer lugar, que la conducta oligopolística era menor en la banca regional, local e industrial, ya que estas entidades presentaban una mayor variedad de tipos que la banca nacional. En segundo lugar, que el denominado «Club de los Siete Grandes» no funcionó bien. Las series de tipos de interés muestran que desde principios de los setenta los bancos de ámbito nacional practicaron políticas propias y no coordinadas.

Una vez hemos constatado la existencia de acuerdos colusivos o monopolísticos para fijar precios por parte de los bancos españoles, la siguiente cuestión sería analizar el grado de efectividad de los mismos. A priori parecería lógico suponer que los bancos no hubieran respetado un tope legal que era tan ajeno a las cambiantes circunstancias económicas al no ser modificado desde 1939 hasta 1964. Para poder obtener evidencia sobre el cumplimiento de dichos acuerdos se han estimado los costes financieros o tipo de interés «efectivo» pagado por los principales bancos españoles (los denominados «cinco grandes bancos») en este período. Para ello dividiremos la cuantía total de costes financieros que aparece en la cuenta de explotación de dichas entidades por el volumen de depósitos captados. El principal problema para estimar el coste efectivo de los depósitos es que en ocasiones los bancos incluían junto con sus costes financieros (es decir, el coste asociado estrictamente al pago de una remuneración por los depósitos) otras partidas, tales como comisiones, e incluso en algunos casos determinadas tasas locales, si bien las Memorias Anuales de los principales bancos indican que el porcentaje que representaban estas partidas era relativamente modesto. Por tanto, la variable que se ha estimado es sólo una aproximación de cuál fue el coste efectivo que pagaron los principales bancos por sus depósitos y será necesario extremar la prudencia a la hora de interpretar los resultados obtenidos. A medida que los bancos permitan acceder a sus archivos será posible obtener información más fidedigna sobre dicha variable.

Una vez obtenido el coste efectivo de los depósitos, se ha calculado cuál sería el coste «máximo» que habrían tenido dichos depósitos si los bancos hubiesen pagado el tipo de interés máximo legal establecido por las autoridades para cada tipo de depósitos (depósitos a la vista, depósitos a plazo de un mes y depósitos a mayores plazos) ${ }^{4}$. A este indicador lo

+ En 1939 las autoridades monetarias fijaron un tipo de interés máximo del 1 por 100 para los depósitos a la vista, del 2 por 100 para los depósitos a corto plazo (a plazo 
denominaremos «coste legal» máximo. Mediante la comparación del coste financiero efectivo pagado por los bancos y del máximo legal es posible establecer una conclusión sobre el cumplimiento de los acuerdos colusivos para fijar los tipos de interés a los que el CSB hace referencia. El principal problema de este indicador es que es una media de los «extra-tipos» pagados por los diferentes tipos de depósitos. Así pues, este indicador no nos permite decir nada sobre las posibles estrategias de los bancos para captar depósitos a corto o a largo plazo. También parece lógico suponer que debió existir una clara asimetría entre pequeños y grandes ahorradores, ya que los bancos sólo pagarían tipos de interés por encima de los legales a sus mejores clientes.

El único banco para el que no se ha podido realizar dicha estimación es el Banco Central, ya que dicha entidad incluye junto con los costes financieros una parte de sus depreciaciones. Así pues, este banco tiene una relación «costes financieros/total depósitos» muy superior a la del resto de entidades por un problema estrictamente contable. El Gráfico 1 muestra los costes financieros para los cuatro grandes bancos (Banco Español de Crédito, Banco Hispano Americano, Banco Vizcaya y Banco Bilbao), mientras que el Gráfico 2 incluye a los cinco grandes bancos (es decir, al Banco Central, pese a los problemas que presenta la estimación del coste efectivo para esta entidad).

El resultado que se obtiene es muy ilustrativo. El Gráfico 1 muestra que sólo hasta 1950 sería posible aceptar que los tres grandes bancos (el Banco Hispano Americano sólo ofrece información de esta variable a partir de 1954) cumplieron los acuerdos sobre los tipos de interés pasivos, ya que entre 1939 y 1950 el coste efectivo de los depósitos fue claramente inferior al máximo legal. Desde este año el coste efectivo de los depósitos se encuentra por encima del máximo legal. El margen entre el «tipo legal» y el «tipo efectivo» se amplía a partir de 1955, y sólo se reduce desde 1966-1967. Por tanto, la evidencia empírica muestra que, si bien existieron acuerdos colusivos para fijar los tipos de interés de las operaciones activas y pasivas, al menos en lo que a las operaciones pasivas se refiere, todo

de un mes) y de un 3 por 100 para los depósitos a plazo de un año. En 1964 se produjo una reducción al 0,5 del tipo de interés de los depósitos a la vista y se estableció un máximo del 4 por 100 para los depósitos de más de dos años. Desde 1967 hay aumentos en los distintos tipos de interés en 1969, 1970, 1973 y 1974, y además desde 1969 (con la excepción del año 1973) se liberalizan los tipos de interés de los depósitos a más de dos años. En el Anuario Económico y Social (Tamames, 1975, p. 373) se ofrece una cuadro resumen de los tipos legales de interés del sistema bancario desde 1939 hasta 1975. 


\section{GRÁFICO 1}

Coste efectivo (CE4) y legal (ML4) de los depósitos, $5 G B$

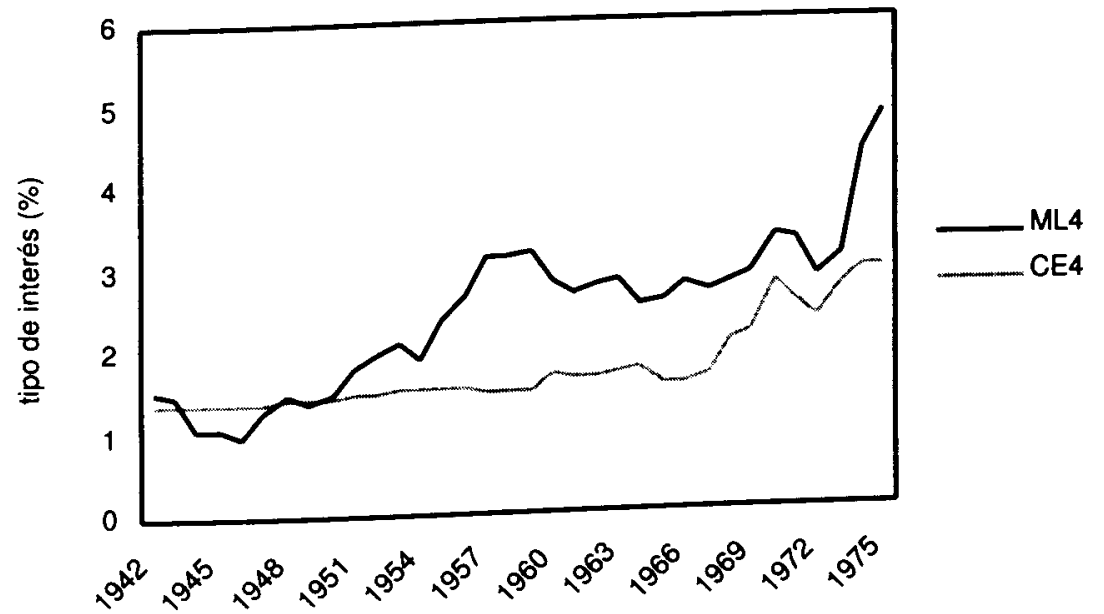

FuENTE: Memorias Anuales de los cinco grandes bancos y Balance Consolidado de la Banca Privada (CSB).

\section{GRÁFICO 2}

Coste efectivo (CE5) y legal (ML5) de los depósitos, 5GB
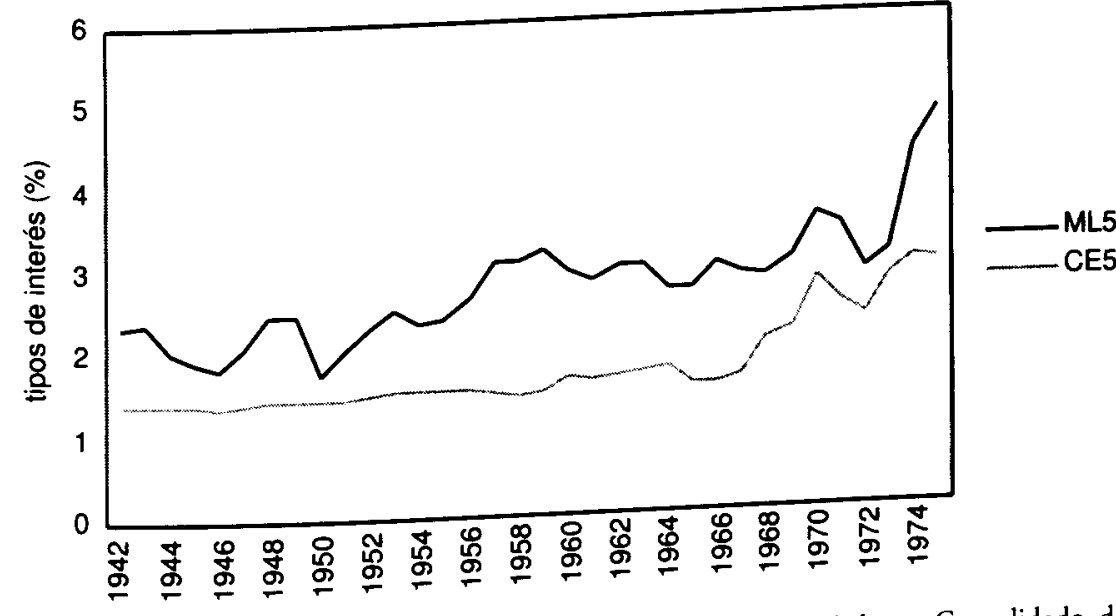

Fuente: Memorias Anuales de los cinco grandes bancos y Balance Consolidado de la Banca Privada (CSB). 
parece indicar que dichos pactos fueron poco efectivos. Los resultados obtenidos muestran que algunos bancos infringieron dichos acuerdos $\mathrm{y}$, en su esfuerzo por captar nuevos depósitos, ofrecieron a sus depositantes remuneraciones por encima del tipo de interés máximo legal (bien mediante el pago de extra-tipos o de otras formas alternativas de remuneración que se incluirían contablemente dentro de esta partida de costes financieros).

$\mathrm{Si}$ analizamos de forma desagregada la información que suministran los cuatro bancos considerados, se obtienen diferencias significativas. El Banco Bilbao entre 1942 y 1950 y el Banco Español de Crédito entre 1942 y 1951 tienen un coste efectivo de los depósitos por debajo del máximo legal. En el caso del Banco Vizcaya este período es más amplio y va desde 1942 hasta 1957. En el caso de los bancos Bilbao y Vizcaya, además, hasta el año 1964 las diferencias entre el coste efectivo de los depósitos y el máximo legal no sólo son pequeñas sino que incluso en algunos años vuelven a tener un coste efectivo de los depósitos inferior al máximo legal. Ésa es la situación, por ejemplo, del Banco Vizcaya entre 1960 y 1963 y del Banco de Bilbao para los años 1953, 1954 y el período 1959-1963. En los casos del Banco Hispano Americano desde 1954 (el primer año para el cual disponemos de información) y del Español de Crédito desde 1951, el coste efectivo de los depósitos está claramente por encima del máximo legal hasta 1975 de forma ininterrumpida.

En resumen, lo que la información desagregada por bancos nos indicaría es que las políticas utilizadas por las principales entidades bancarias en lo que a fijación de tipos de interés para captar el pasivo fueron diferentes. En ese sentido, sí que parece observarse un cierta competencia vía precios.

Un informe del Foreign Office de 1954 escrito por Macgilliaray sobre el sistema bancario español y enviado al Banco de Inglaterra (Archivos del Banco de Inglaterra, 1954) ofrece información cualitativa sobre la posible existencia de competencia a través de los tipos de interés entre los bancos españoles:

«Aunque en teoría existe un pacto de caballeros entre los bancos sobre los tipos de interés, se le presta poca atención y hay bastante competencia. El Banco de Londres y Sudamérica, que parece ser el único que continúa adhiriéndose a dicho acuerdo, ha perdido una parte considerable de sus clientes como consecuencia de que continúa pagando sólo el tipo de interés de los depósitos del $1 / 2$ por 100 que se había acordado, una tasa muy por debajo de la que están pagando el resto de bancos» 5 .

\footnotetext{
5 Traducción propia.
} 
En un informe de 14 de abril de 1962 aparece un comentario similar: «Dado el poco realista tipo de interés del $1 / 2$ por 100 que ha sido fijado para los depósitos a la vista, y en ausencia de sanciones reales en contra de los que infringen el pacto, es más que probable que la mayoría de bancos lo abandonen.»

Del mismo modo, Tortella y García Ruiz (1999) en su estudio, todavía sin publicar, sobre los bancos Central e Hispano Americano indican que la posición de ambas entidades frente a los acuerdos para fijar los tipos de interés era muy diferente. Por ejemplo, según estos autores, el Banco Central siempre fue más partidario de mantenerse dentro de los acuerdos y no pagar extra tipos. El Banco Hispano Americano mantuvo, sin embargo, una postura muy distinta. De hecho esta entidad se sale de la Ordenación Reguladora de Condiciones Mínimas en 1949, siendo las cantidades que se computan como pago de extra-tipos entre 1949 y 1953 relativamente elevadas. Entre 1960 y 1974 no hay información sobre las cantidades que dicho banco destinó al pago de «extra-tipos», excepto en algunos años de forma esporádica. A partir de 1974 el banco vuelve a ofrecer información sobre el pago de «intereses diferenciales» o «intereses elevados», y las cantidades que se incluyen en estas partidas son muy elevadas. De igual modo, Martínez Méndez (1975), que se encontraba en esos momentos en el Servicio de Estudios del Banco de España, reconocía en 1975 la importancia que tenía en España el pago de «extra-tipos» en la industria bancaria. Pese a la evidencia mostrada, el CSB nunca aceptó la existencia de «extra-tipos», y en innumerables ocasiones insistía en que ésta no era una práctica general en nuestro sistema bancario ${ }^{6}$.

La principal conclusión que se obtiene es que pese a los intentos por mantener acuerdos de colusión, desde principios de los cincuenta los bancos compitieron vía precios en el mercado de captación de pasivos. Entre 1942 y 1950 es difícil pensar que los bancos españoles se vieran en la necesidad de utilizar otras formas de remuneración alternativas al tipo de interés, ya que ni tan siquiera pagaban el máximo legal. La situación, sin embargo, cambia en 1950. A partir de este año algunos bancos españoles (Banco Español de Crédito y Banco Hispano Americano, de forma ininterrumpida, y Banco Bilbao y Banco Vizcaya, en momentos más puntuales) tuvieron un coste de sus depósitos por encima del tipo de interés legal. Este hecho

- Dicha conclusión se puede extraer de la lectura de las Circulares emitidas por dicha institución. 


\section{GRÁFICO 3}

Desviación estándar del coste efectivo de los depósitos

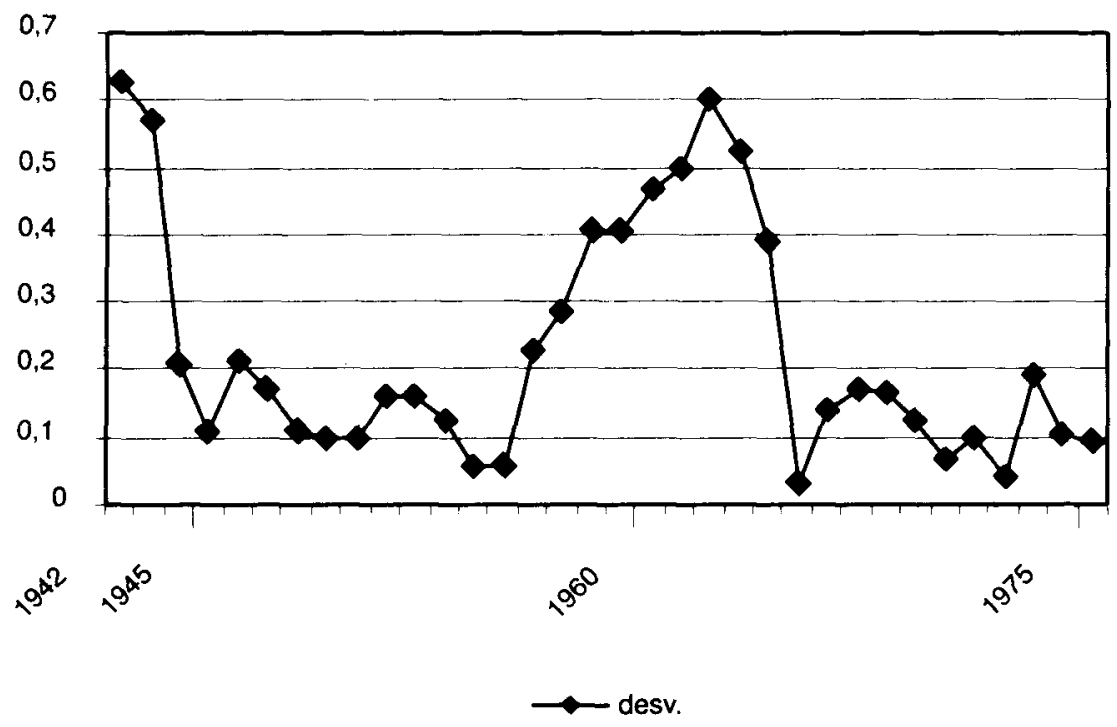

FUENTE: Ver gráficos 1 y 2.

indicaría dos cosas. En primer lugar, que desde mediados de los cincuenta existió una cierta competencia entre los principales bancos españoles para captar depósitos. En segundo lugar, que dentro de los costes financieros los bancos pudieron estar incluyendo otra serie de costes adicionales al tipo de interés legal necesarios para captar depósitos. El aumento más espectacular del coste «efectivo» de los depósitos en relación al coste «legal» máximo se produce a partir de 1957.

Evidentemente, que los bancos paguen «extra-tipos», no necesariamente implica competencia. Es posible que los principales bancos adoptasen un acuerdo colusivo que implicase el pago de unos tipos de interés para los depósitos superiores al legal. Sin embargo, es el hecho de que no todos los bancos adoptasen la misma estrategia lo que permite aceptar algún grado de competencia en el mercado de los depósitos. El Gráfico 3 muestra la dispersión entre el coste efectivo de los depósitos de los cinco grandes bancos. Como puede observarse, desde 1942 hasta 1955 el nivel de dispersión entre el coste efectivo de los depósitos de los cinco grandes bancos es muy bajo. Sin embargo, esta dispersión aumenta de forma importante 
desde 1955 hasta 1965. Probablemente es en este período cuando los bancos trataron de utilizar los tipos de interés como un instrumento para captar depósitos. A partir de 1965 esta dispersión vuelve a disminuir, lo que podría indicar una vuelta a los acuerdos colusivos por parte de los principales bancos. Es curioso observar cómo es precisamente desde 1962, año en el que, tal y como señalamos anteriormente, Ignacio Villalonga comienza a reunir semanalmente a los principales banqueros, cuando se inicia la caída de la dispersión.

Sería muy interesante realizar una estimación similar del tipo de interés que los bancos cobraban por sus préstamos, sin embargo, en este caso las dificultades son todavía mayores que por el lado de los depósitos. La mayoría de los bancos no diferenciaba entre los ingresos procedentes de su actividad crediticia y los que provenían de otras inversiones alternativas. La única entidad que diferencia, desde 1951, entre: $a$ ) los «ingresos procedentes de la explotación normal», y $b$ ) los «beneficios procedentes de la cuenta de valores», es el Banco Español de Crédito. Este banco obtendría una tasa de remuneración sobre sus créditos (si es que esta estimación puede servir como una aproximación al tipo de interés que cobraban por los créditos) muy elevada, que parte del 14 por 100 en los años cincuenta para alcanzar un máximo del 30 por 100 en 1967. Es difícil pensar que los bancos españoles estuviesen cobrando por sus préstamos unos tipos de interés tan elevados y, por tanto, las conclusiones por el lado del activo son muy débiles. De momento no hay información alternativa que nos permita conocer cuáles fueron los tipos de interés que tuvieron que pagar las empresas españolas por sus créditos. Aunque existen algunas referencias a las importantes cargas financieras que tuvieron que soportar algunas empresas españolas en este período (Antolín, 1996), de momento la información disponible sobre la política de tipos de interés activos adoptada por los principales bancos españoles es todavía más escasa que sobre la captación de pasivo.

Tras obtener evidencia sobre una posible competencia entre las principales entidades bancarias vía precios, se examinará en qué medida dicha competencia tuvo algún efecto en el mercado de depósitos, es decir, si los bancos más agresivos en precios fueron capaces de captar un mayor volumen de depósitos. La cantidad de depósitos captada por una entidad bancaria depende de un conjunto de variables entre las que destacaríamos: el tipo de interés y el número de oficinas. Cuando la competencia vía precios está regulada, los bancos utilizan la proximidad geográfica como una forma de remuneración para sus clientes. Sin embargo, algunos autores 


\section{GRÁFICO 4}

Relación entre Depósitos y Coste Efectivo de los mismos

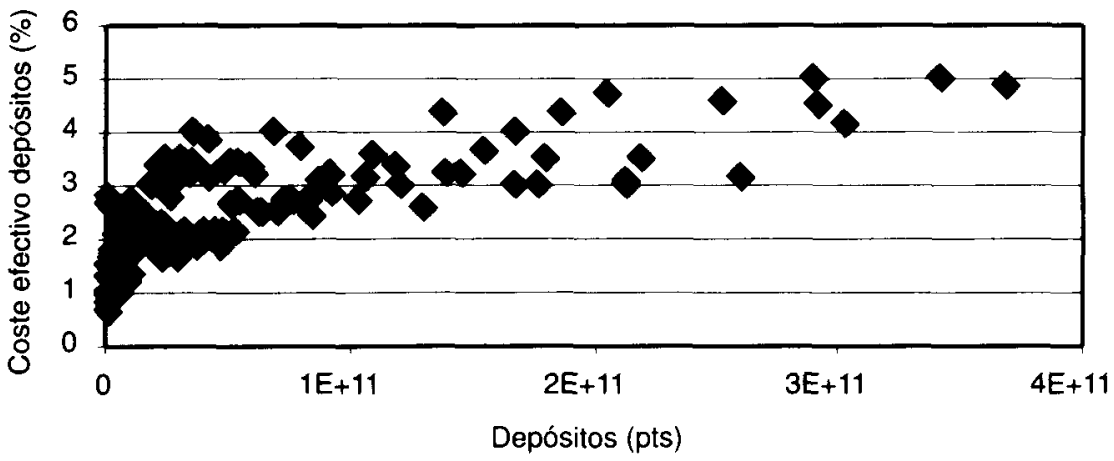

Fuente: Memorias Anuales del Banco Bilbao, Central, Español de Crédito y Vizcaya y Balances Consolidados de la Banca Privada (CSB).

\section{GRÁFICO 5}

Relación aumento Depósitos y extra-tipos

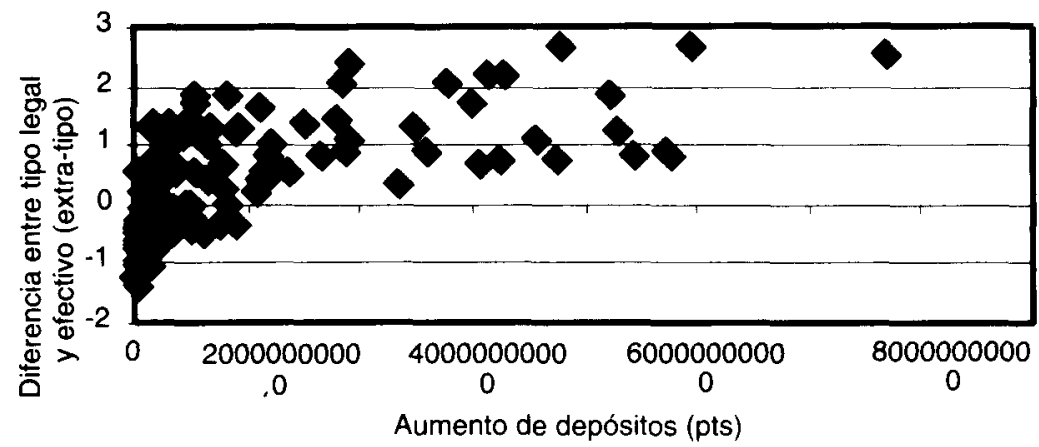

Fuent7: Memorias Anuales del Banco Bilbao, Central, Español de Crédito y Vizcaya y Balances Consolidados de la Banca Privada (CSB).

consideran que existen otra serie de variables sociales y psicológicas tales como la edad, educación, nivel social, entre otras, que son esenciales de cara a explicar por qué un individuo escoge un determinado tipo de entidad financiera para depositar sus ahorros (Coello, 1998). Por tanto, para analizar la incidencia del tipo de interés en la captación de depósitos para las distintas entidades bancarias, deberíamos incluir otra serie de variables 
(número de oficinas, competencia del resto de entidades financieras, nivel de desarrollo, etc.) para las que es difícil obtener información desagregada para este período. Por tanto, nos limitaremos a ver si existe una relación entre el tipo de interés que los bancos pagaban por sus depósitos y el volumen captado de los mismos (Gráfico 4). De igual modo se analizará la conexión existente entre el pago de «extra-tipos» (medido como la diferencia entre el tipo de interés efectivo y el tipo legal) y el aumento en el volumen de depósitos de dichas entidades para los años 1945, 1950, $1955,1960,1965,1970$ y 1975. Los resultados se muestran en el Gráfico 5.

En el Gráfico 4 aparecen todas las observaciones disponibles para el período 1942-1975 de volumen de depósitos y coste efectivo de los mismos. Se observa cómo un mayor coste efectivo de los depósitos va asociado, en la mayoría de los casos, a un mayor volumen de depósitos. Sin embargo,

\section{GRÁFICO 6}

Relación entre el aumento de los depósitos y el «extra-tipa» para los cuatros grandes bancos (Banco Bilbao, Banco Español de Crédito, Banco Hispano Americano y Banco Vizcaya)

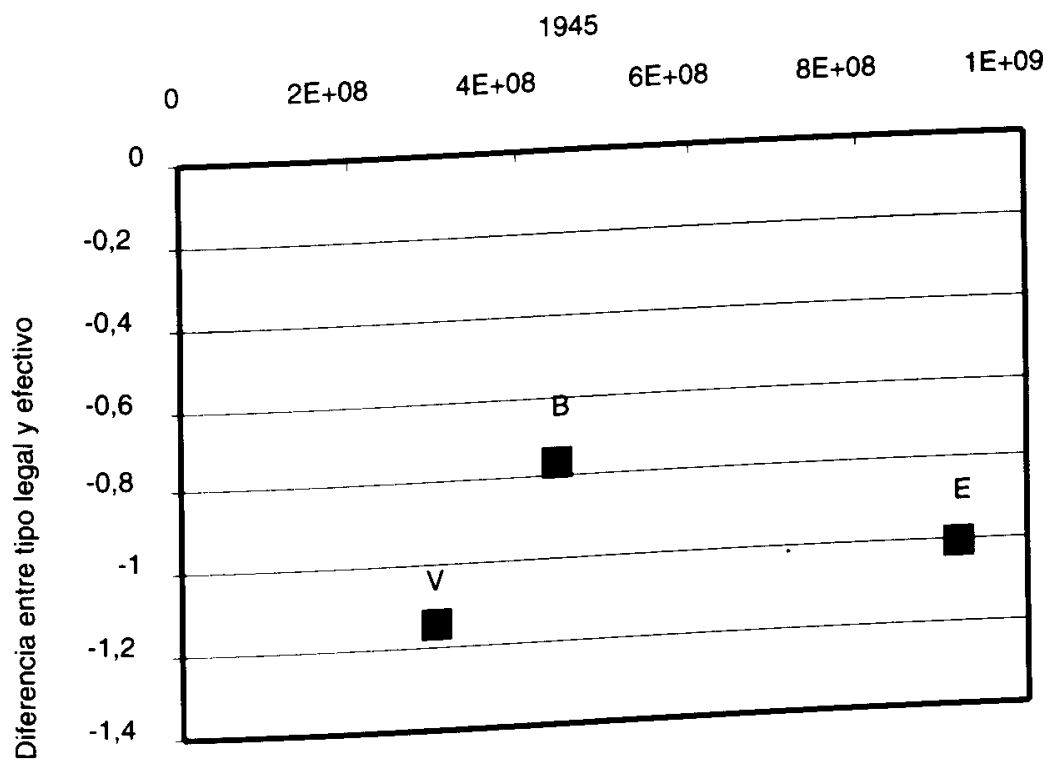

Aumento depósitos 
1950

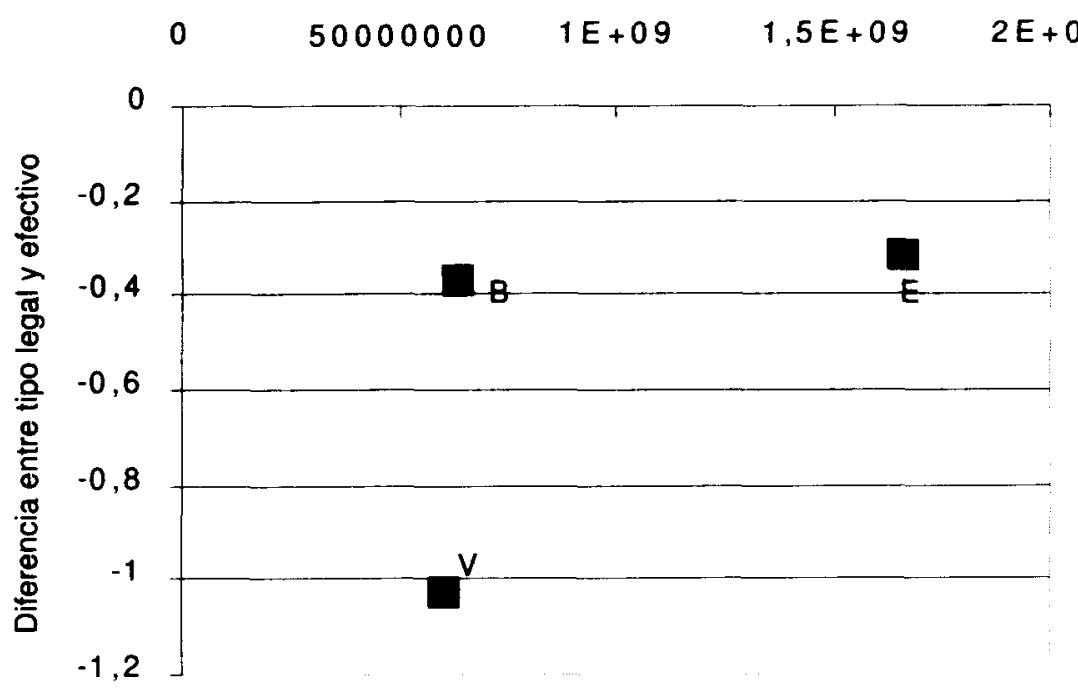

Aumento depósitos

1955

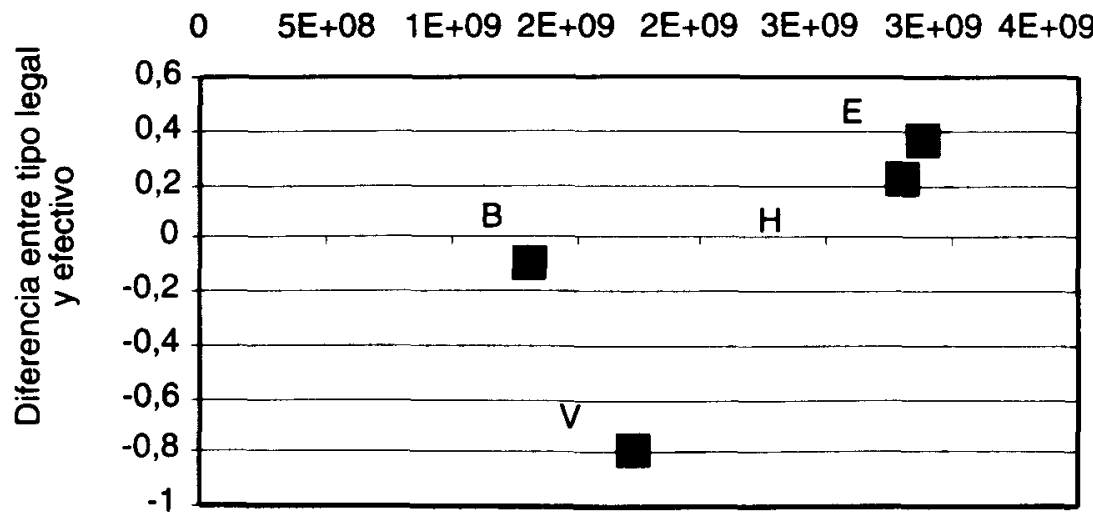

Aumento depósitos 


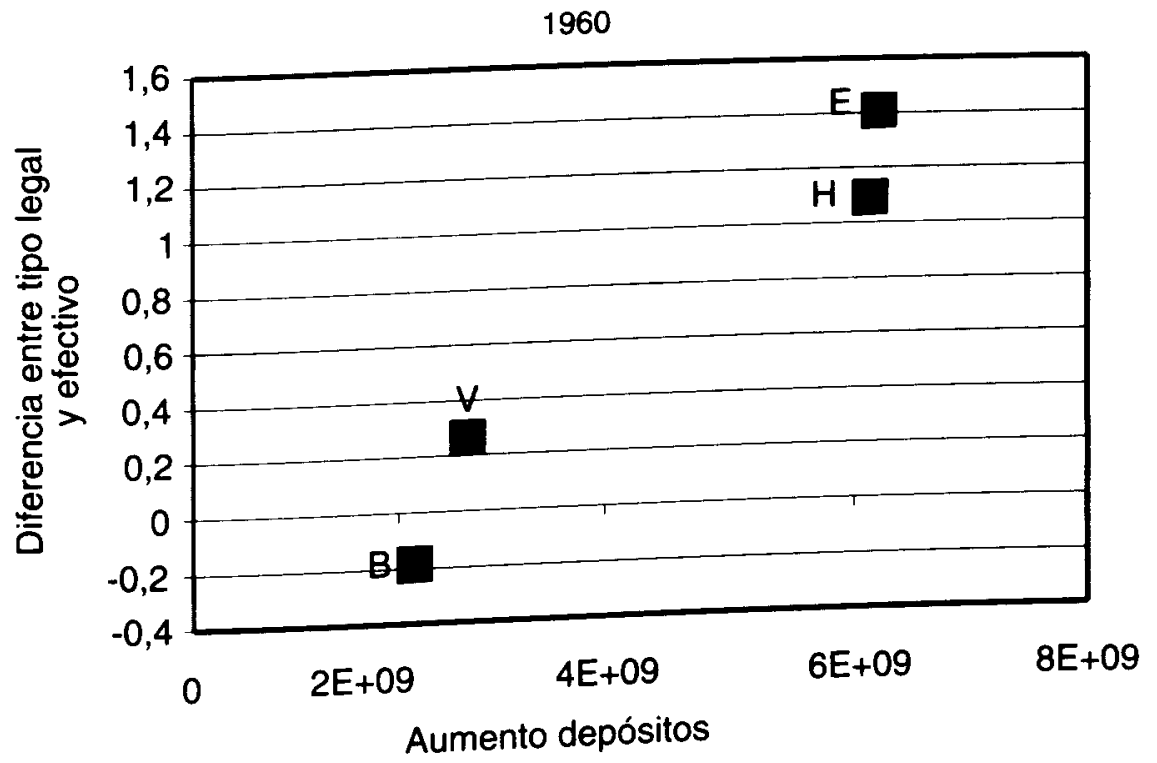

1965

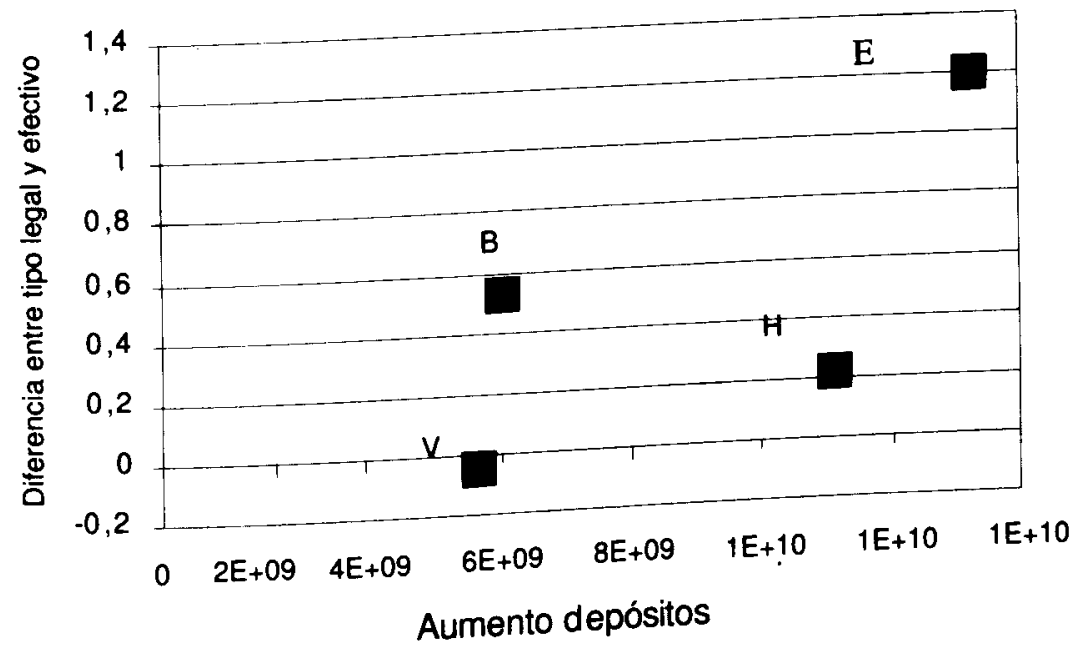


1970

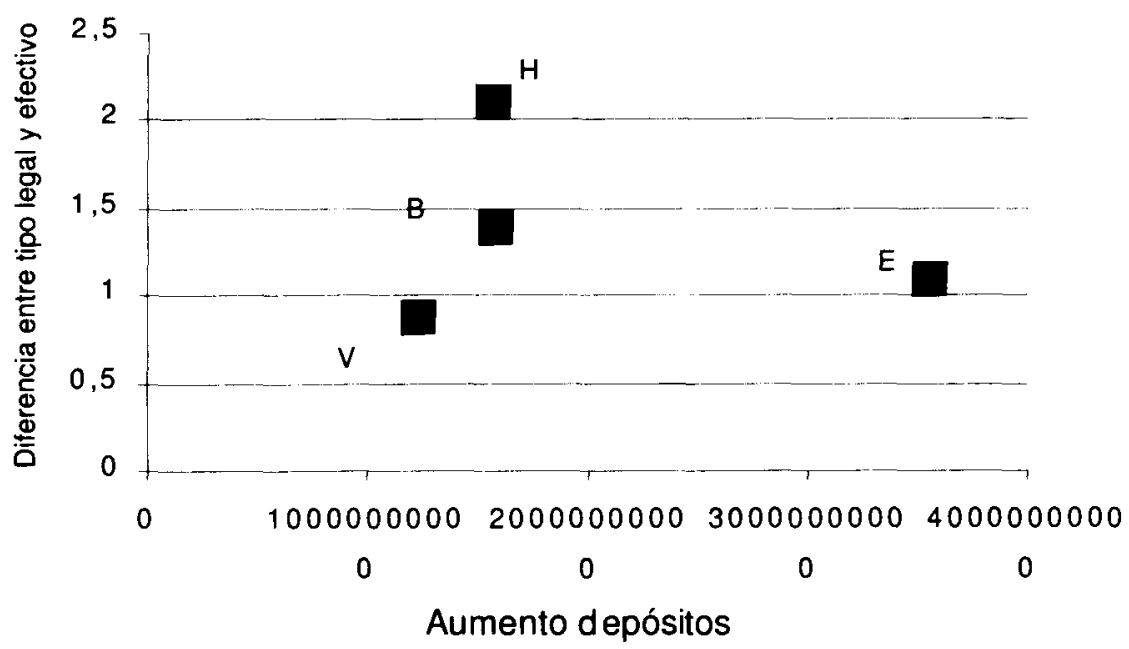

1975

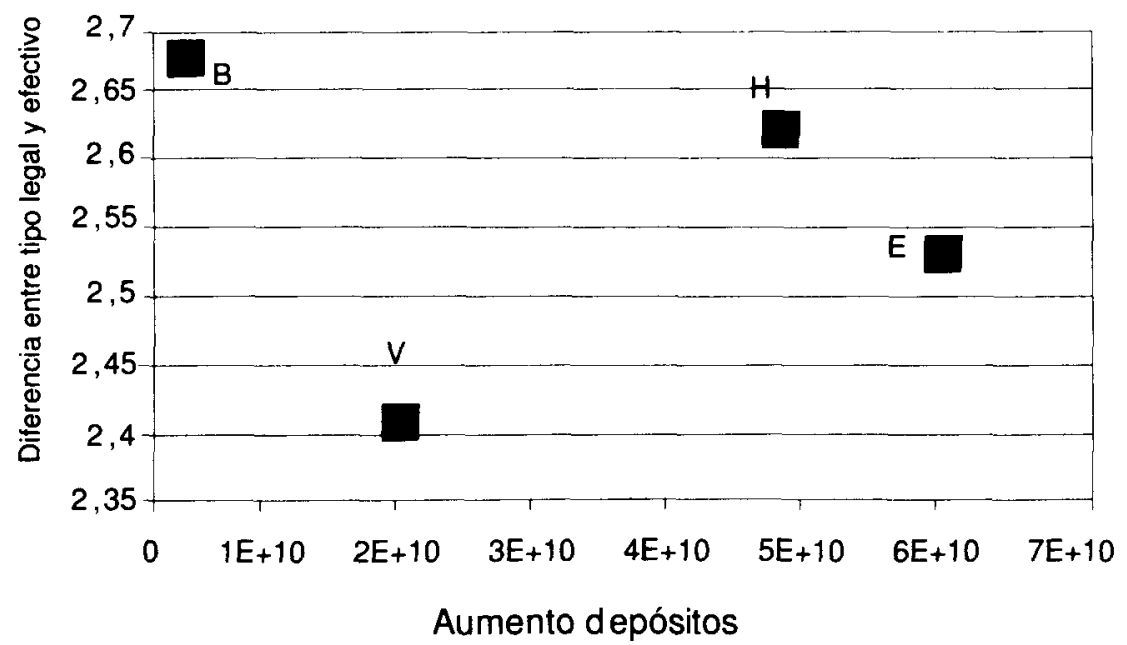

FuENTE: Memorias Anuales de los grandes bancos y Balances Consolidados de la Banca Privada (CSB). 
para ver si el esfuerzo que hacían los distintos bancos pagando una remuneración más elevada («extra-tipo») se reflejaba en un mayor aumento de sus depósitos hemos realizado los Gráficos 5 y 6 . En el Gráfico 5 se muestran todas las observaciones disponibles para el período 1942-1975 de aumento en los depósitos con respecto al año anterior, así como del «extra-tipo» que pagaban por sus depósitos las principales entidades bancarias. En el Gráfico 6 se obtiene la relación entre ambas variables para los cuatro grandes bancos en los años 1945, 1950, 1955, 1960, 1965, 1970 y 1975.

El Gráfico 5 ofrece una imagen similar a la del Gráfico 4. En general, los mayores aumentos en el volumen de depósitos se corresponden con «extra-tipos» más elevados. Para ver con mayor detalle cuál era esta relación para los bancos analizados hemos realizado el Gráfico 6. Este gráfico muestra que en los años 1950, 1955 (con la excepción del Banco Vizcaya), 1960, 1965 (con la excepción del Banco Hispano Americano) y 1970 (.con la excepción del Banco Español de Crédito) hay una relación entre los bancos que pagaron mayores «extra-tipos» y los bancos que consiguieron un mayor aumento en sus depósitos. Dicha relación no se cumple ni para todos los años ni para todos los bancos. Sin embargo, como ya he señalado anteriormente, es necesario tener en cuenta que hay otro conjunto de variables, especialmente el número de oficinas, que puede ser tan importante al analizar el aumento de depósitos conseguido por una determinada entidad bancaria como el tipo de interés, especialmente a partir de 1962, cuando el número de oficinas se convierte en un instrumento esencial para competir por los depósitos.

\section{CONCLUSIONES}

Desafortunadamente, no hay un modelo de competencia imperfecta en el sector bancario que permita analizar de forma precisa el funcionamiento del mismo (Repullo, 1990). Esto ha llevado a la utilización de los denominados modelos de «estructura-conducta-funcionamiento» que utilizan el nivel de concentración para medir el grado de competencia de la banca. De ese modo, los diversos índices de concentración, la rentabilidad y las conexiones entre las entidades bancarias, así como entre los bancos y las principales empresas industriales, han sido las variables que preferentemente se han utilizado para definir al sector bancario español como un sector monopolista. Las críticas ha esta literatura son fundamentalmente 
dos. El primer conjunto de críticas estaría relacionado con los problemas derivados de utilizar el nivel de concentración como indicador del poder de monopolio de un sector. A través del índice de concentración no es posible diferenciar entre la hipótesis de poder de mercado y la hipótesis de eficiencia, según la cual un mayor nivel de concentración podría ser el resultado de la existencia de economías de escala y no de un problema de colusión (Smirlock, 1985). El segundo grupo de críticas hace referencia a los resultados que se obtienen del análisis del nivel de concentración y rentabilidad del sector bancario español. Tanto para el conjunto del sector como para los diferentes grupos bancarios se observa una disminución de los índices de concentración desde 1954. Tampoco hay diferencias significativas que permitan afirmar que la rentabilidad de los bancos españoles estuvo muy por encima de la rentabilidad de las empresas no financieras. Por tanto, es necesario utilizar otras variables que ofrezcan información más precisa sobre las prácticas bancarias si queremos conocer cuál fue el funcionamiento y la estructura del sector bancario español desde el final de la guerra civil hasta mediados de los setenta.

Con ese objetivo, en este trabajo se ha analizado la conducta de los principales bancos españoles en lo que a fijación de precios se refiere. Tal y como se ha mostrado en la sección 2 , el marco legislativo existente permitía a los bancos llegar a acuerdos monopolistas para fijar los tipos de interés de los depósitos y los créditos. El CSB afirma que los bancos españoles establecieron un pacto por el que se comprometieron a pagar un tipo de interés sobre sus depósitos por debajo del máximo legal y a cobrar un tipo de interés sobre sus créditos por encima del mínimo legal. La evidencia cualitativa y cuantitativa que se presenta en este trabajo muestra que dichos pactos fueron poco efectivos, al menos durante una parte del período estudiado. Diversos informes sobre el funcionamiento del sistema bancario en ese periodo insisten en el pago de «extra-tipos» por parte de los bancos españoles. De igual modo, la estimación realizada de los costes financieros para las principales entidades bancarias permite obtener tres conclusiones. En primer lugar, desde principios de los cincuenta el coste efectivo de los depósitos para algunos de los principales bancos españoles tales como el Español de Crédito o el Hispano Americano estuvo por encima del máximo legal. En segundo lugar, hay diferencias significativas entre el coste financiero de los bancos utilizados en este estudio, lo que difícilmente permitiría aceptar que dichas entidades se mantuvieron dentro de un acuerdo colusivo para fijar sus tipos de interés. La evolución de las tasas de dispersión del coste efectivo de los depósitos muestra una 
muy baja dispersión desde 1945 hasta 1955, un aumento significativo de la misma (y, por tanto, una menor probabilidad de que los bancos se mantuviesen dentro de los acuerdos colusivos) entre 1955 y 1965, y finalmente una caída sustancial de la dispersión a partir de 1965 (lo cual podría mostrar un intento por parte de los cinco grande bancos de volver a fijar acuerdos colusivos sobre los tipos de interés de los depósitos). Finalmente, aunque hay algunas excepciones, los bancos que pagaron una mayor remuneración sobre sus depósitos consiguieron captar un mayor volumen de los mismos, lo cual mostraría una cierta efectividad de la competencia ejercida entre las distintas entidades para atraer pasivo.

La principal conclusión que se obtiene es que en el sector bancario español desde principios de los cincuenta existió una cierta tensión entre colusión y competencia y que en algunos momentos la competencia vía precios (imperfecta, sin duda) dominó sobre los acuerdos restrictivos de la competencia. Pese a ello, es evidente que los grandes bancos trataron, en la medida de lo posible, de huir de la competencia, y eso explicaría la importante caída de la tasa de dispersión del coste efectivo de los depósitos a partir de 1962, y especialmente tras 1965.

Pese a la existencia de «extra-tipos», y aunque en algunos momentos los principales bancos españoles trataron de utilizar los precios como un mecanismo para atraer depósitos (probablemente no para todos sus clientes sino para los que depositaban cantidades elevadas), es evidente que la regulación fue un mecanismo útil para mantener el tipo de interés medio sobre los depósitos por debajo del tipo de interés de mercado. Dadas las elevadas tasas de inflación, el tipo de interés real medio no sólo era muy bajo sino que incluso fue negativo en algunos años. Por tanto, el hecho de que obtengamos evidencia de la existencia de «extra-tipos» e incluso de que aceptemos un cierto nivel de competencia en una parte del período estudiado no altera de forma sustancial los efectos negativos que la regulación financiera pudo tener en la captación de ahorro por parte del sistema financiero español.

\section{BIBLIOGRAFIA}

\section{Fuentes no impresas}

Archivo Historico del Banco de Espana (1946a): Informe del Consejo Superior Bancario sobre el Proyecto de Ley de Ordenación Bancaria de 1946, sin catalogar. 
Archivo Histórlco del Banco de España (1946b): Informe del Banco de Vizcaya sobre el Proyecto de Ley de Ordenación Bancaria de 1946, sin catalogar.

ARCHIVO DEL BANCO DE INGLATERRA (1954): Report of the Overseas and Foreign Officce, written by Macgilliary, OV61/5, 21 may, p. 11.

- (1962): Report of 14 April, OV61/7, p. 8.

\section{Fuentes impresas}

Antolin, Francesca (1996): «Hidroeléctrica Ibérica y la electrificación del País Vasco», en Comin, Francisco, y Martin Aceña, Pablo, La empresa en la bistoria de España, Madrid, Civitas, pp. 237-264.

Caminal, Ramón; Gual, Jordi, y Vives, Xavier (1990): «Competition in Spanish banking», en Dermine, J. (ed.) (1990): European Banking in the 1990's, Oxford, Basil Blackwell, pp. 271-321.

Consejo Superior Bancario (1942-1975): Balances de la Banca Privada.

- (1976-1982): Recopilación de Circulares.

DE LA SIERRA, Fermín (1953): La concentración económica de las industrias básicas españolas, Madrid, Instituto de Estudios Políticos.

EDWARDS, Jeremy, y FISCIIER, Klaus (1994): Banks, finance and investment in Ger many, Cambridge, Cambridge University Press.

Fanjul, Óscar, y Maravall, Fernando (1985): La eficiencia del sistema financiero español, Madrid, Alianza Editorial.

Martinez Méndez, Pedro (1990): Los beneficios de la banca privada, 1970-1989, Banco de España, Madrid.

Memorias: Banco de Bilbao, Banco Central, Banco Hispano Americano y Banco de Vizcaya, 1939-1975.

Muñoz, Juan (1969): El poder de la banca en España, Madrid, Zero.

NeEdham, Douglas (1978): The economics of Industrial Structure-Conduct and Performance, London, Holt, Rinehart \& Wiston.

Pons, M. A Angeles (1999): «Capture or agreement? Why Spanish banking was regulated under the Franco regime (1939-1975)», Financial History Review, 6, 1, april, pp. 25-46.

- (2001): Regulating Spanish Banking, 1939-1975, Ashagate. En prensa.

PuEYo, Javier (1998): «La concentración económica de la banca privada en España en el siglo $\mathrm{xX}$ ». Versión corregida de la ponencia con el mismo título presentada en el VI Congreso de la Asociación de Historia Económica en Gerona.

Repulio, Rafael (1990): «Comentario a "Competition in Spanish banking"», en Dermine, Jean (ed.) (1990): European Banking in the 1990's, Oxford, Basil Blackwell, pp. 322-324.

SMiRLock, Michel (1985): «Evidence and (non)relationship between concentration and profability on Banking», Journal of Money, Credit and Banking, 17 (1), february, pp. 69-83.

TaFunfll, Xavier (1998): «Los beneficios empresariales en España, 1880-1981. Una estimación de un índice anual del excedente de las grandes empresas», Revista de Historia Económica, núm. 16 (3), pp. 707-746.

Tamames, Ramón (ed.) (1975): Anuario Económico y Social de España, Barcelona, Ancesa, S. A. 
Tortella, Gabriel, y García Ruiz, Jose Luis (1999): «Una historia de los Bancos Central e Hispano Americano, 1900-2000: Un siglo de gran banca en España», texto mecanografiado sin publicar (realizado con fuentes externas e internas).

- (1993): «Una historia de los Bancos Central e Hispano Americano», texto mecanografiado sin publicar (realizado con fuentes externas).

WeIss, Leonard W. (ed.) (1991): Structure, Conduct and Performance, London, Haverton Wheatsheaf. 